\title{
Geoliteracy, cartology, and a mobile serious game
}

\author{
Yaïves Ferland $^{\mathrm{a}, *}$, Margot Kaszap ${ }^{\mathrm{b}}$ \\ ${ }^{a}$ Université Laval, Québec, Canada, yaives.ferland@scg.ulaval.ca \\ ${ }^{b}$ Université Laval, Québec, Canada, margot.kaszap@fse.ulaval.ca \\ * Corresponding author
}

Keywords: Geographic literacy, Cartology, Experiential learning, Learning threshold, Mobile serious game trajectories

\begin{abstract}
:
Some actual research teams in Education Science go toward the development of educative serious games on mobile devices for letting elementary school pupils (i.e. primary school students) playing outdoor to learn geographic facts, concepts, and patterns. The challenge is about improving their geographic literacy and fluency, or 'geoliteracy', and their map-reading competencies, called 'cartology', before their adolescence as critical development 'threshold'.

The aspects one has to work on consider the ways to learn, use, and comprehend maps as geospatial representations, both concrete display of a terrain on the paper sheet or on a digital screen and, on another hand, cognitive configuration in the mind that structures, interprets, and recalls on demand geospatial information on location or orientation at geographical scales. The fundamental interest of cartographic abilities to make and read a map is that it creates information value, structures memory about places and events, and enhances mobility.

In fact, there is a societal concern that a majority of adult population is not geographically literate neither efficient in reading and using maps in real-life context, even for just path finding. The main concern to address early at school is still "why" and "how to repair that situation"? If their geospatial cognitive development was weak at school, then that impedes them to comprehend geospatial concepts, structures, and information, later as adults. If a student does not succeed to pass over a kind of learning threshold, even the few abilities feebly acquired may vanish without significant usage neither interest in them. Later, it will be very hard to restart learning of that same matter without the necessary mental frames to organize geographical concepts and relations into an actionable knowledge.
\end{abstract}

Facing this geoliteracy challenge, the geographical map appears as the best, powerful, and necessary support or instrument of geospatial knowledge representation. One may define geoliteracy as a set of stabilized and adaptive cognitive abilities and functional competencies to handle, by self, geographical realities and cartographical representations. According to Edelson (2012), the three components of achieved geoliteracy are to develop consciousness of geographical interactions (understanding of human and natural systems in space), interconnections (geographic reasoning), and implications (systematic decision-making).

Thus in detail, a geoliterate adult should develop abilities in geospatial thinking and possess a complete (but rarely exhaustive) set of skills that are necessarily useful in normal autonomous life to:

- read, use, and even detect errors on maps and other carto-geographic representations (at any format, support, and scale or zoom level);

- locate places and situations occurring here and there, find new ways in space (at any scales);

- understand and interpret geospatial concepts, signs, and structures on a critical, reasoned, and wise fashion, while discarding misconceptions;

- determine, delimit, plan, and select best places to install activities;

- recall modes and patterns of geospatial (not only geometrical neither topological) representation, even without maps at hand (not just from mental images, capital cities, touristic metaphors, or evocative pictures to come out from memory, which is necessary, of course, but not sufficient);

- enhance own geographic culture, multiscale perspective, and useful geospatial awareness;

- elaborate an opinion or explanation regarding daily geospatial situations or circumstances.

What a troubling concern is the multiple evidences that the majority of adult population is not literate neither efficient in just reading and using maps, i.e. cannot perform most of the precedent list of geospatial abilities and competencies. 
A research team joined with elementary schoolteachers, within a small community of practice, in order to identify pedagogic needs and test some game components as exercises in class context; then emerged the project Géolittératie (2015-2017). The pedagogic goal in designing an educative serious game on mobile device is to apply conceptual and applied methods for both learning and teaching geospatial competencies accordingly to the official school curriculum. That requires theoretical and methodological considerations about educative serious game (Kaufman \& Sauvé, 2010), cartographical semiology (Bertin, 1967, 1983), the four cognitive development stages for geospatial representation by children (Piaget, 1967), and the experiential learning cycle model (Kolb, 1978, 1984). This kind of cycle supports Piaget's learning phases, from topologic perception to spatial conceptualisation, as well as the three main cartographic processes of map-making, reflexive visualization, and map-reading, which sustain any geographical reasoning.

A methodological framework of a mobile serious game was designed didactically with maps and other components following an increasing complexity, step by step of play. The teacher has to prepare a sequence of tasks to perform in a progressive game according to the different learning styles, for exposing practically the pupils to the cartographical process of making a plan, then a map to use thereafter. Students should like going outdoor on the terrain to gather data in order to answer a question on a theme of investigation related to a curriculum matter. They will consider a designed scenario of typical steps (or "rounds"), within a geospatial environment, that tells a progressive plot and the rules of the game. Thus, they will choice and follow different types of geometrical and geospatial trajectories, that lead the story toward the goal of the game, while taking field-notes on their way as answering questions of the scenario. Then, they draw their collected data on a plan or map and explain in conclusion what happened to the story (and what they learn) due to the spatial organisation of the site or area.

Progress in complexity levels of scenario may start with choosing between right or left to reach the next point of interest, to trying to plan both the shortest and the more pleasant paths to visit the spots where to settle a youth club in the neighbourhood. Types of trajectories going from place to place, in increasing complexity as the rounds of game advance, are based on geometrical primitives: point, succession of points, line, side of line, polyline, polygon, network, open surface, limited surface.

The pedagogic result encompasses both concrete display of a terrain (on paper or on a screen) and learned cognitive configurations in the mind. Only such mental or cognitive representations allow structuring, interpreting, and recalling on demand from memory geospatial information on location, distance, or orientation, within a situation that occurs at geographical scales. Therefore, for these pupils, the fundamental question in geography shall no more be "where" but "how and why is this situation there?"

At that point, only the first half of the experiential learning cycle is accomplished and the cognitive development process be achieved just at the phase associated to a threshold of operational comprehension. Now, the students know how to describe a spatial situation and to make a map, good but not enough. The challenge remains to learn from this quite technical knowledge how to deeply read a map, any map, and to get dense information from it; it is a reflexive, analytical, abstract new phase called visualization.

That phase engages a second process along the second half of the experiential learning cycle, which mirror or complement the cartographic one: a cartological process. A definition for cartology could say "to make the map talking", even for telling a new story. Since player students now know the characteristics of a map, its cartographic "alphabet" composed of dimensions, scale, extent, and semiological symbols, the way is open to ask question by self to the map. They can read on it information that even the map-maker did not know neither put on it, project the map over the place represented and make a wise decision for planning or travelling. One can organize the steps of the cartologic process into another mobile game with scenarios and trajectories for gaining a better understanding of the power of maps for the cognitive structuration of geographical space and learn more efficiently about a specified theme that, for instance, composes historical thought and geographical reasoning about that place. A good theme to begin with is about the meaning of the toponymy in the neighbourhood.

A prototype mixes these mobile serious game components (map, theme, scenario, and trajectory) into a scheme of about fifteen successive rounds of play, then engaging the abilities relative to the three main cartographic processes, along a complete experiential cycle. Part of this method for developing geoliteracy by combination of both cartography and cartology within a serious game was tested recently with undergraduate students in didactic course. Practical experiments must continue strengthen the theoretical and methodological frame and ease the schoolteacher's work in the best usage of maps to structure the geographical comprehension of home place and the World. 